\title{
Thermal conductivity and torsional oscillations of solid ${ }^{4} \mathrm{He}$
}

\author{
M.Yu. Brazhnikov ${ }^{1,2}$, D.E. Zmeev ${ }^{1,3}$, and A.I. Golov ${ }^{1}$ \\ ${ }^{1}$ School of Physics and Astronomy, The University of Manchester, Manchester M13 9PL, UK \\ E-mail: golov@man.ac.uk \\ ${ }^{2}$ Institute of Solid State Physics RAS, Chernogolovka, Moscow Region 142432, Russia \\ ${ }^{3}$ Department of Physics, Lancaster University, Lancaster LA1 4YB, UK
}

Received August 10, 2012

\begin{abstract}
Polycrystalline samples of hcp ${ }^{4} \mathrm{He}$ of molar volume $V_{m}=19.5 \mathrm{~cm}^{3}$ with small amount of ${ }^{3} \mathrm{He}$ impurities were grown in an annular container by the blocked-capillary method. Three concentrations of ${ }^{3} \mathrm{He}, x_{3}$, were studied: isotopically purified ${ }^{4} \mathrm{He}$ with the estimated $x_{3}<10^{-10}$, commercial 'well-grade' helium with $x_{3} \sim 3 \cdot 10^{-7}$ and a mixture with $x_{3}=2.5 \cdot 10^{-6}$. Torsional oscillations at two frequencies, 132.5 and $853.6 \mathrm{~Hz}$, and thermal conductivity were investigated before and after annealing. The solid helium under investigation was located not only in the annular container but also in the axial fill line inside two torsion rods and dummy bob of the doublefrequency torsional oscillator. The analysis of the frequency shifts upon loading with helium and changing temperatures of different parts of the oscillator suggests that the three techniques probe the properties of solid helium in three different locations: the two different torsion modes respond to the changes of the shear modulus of solid helium in either of the two torsion rods while the thermal conductivity probes the phonon mean free path in solid helium inside the annular container. The temperature and width of the torsional anomaly increase with increasing frequency and $x_{3}$. The phonon mean free path increases with increasing $x_{3}$. Annealing typically resulted in an increased phonon mean free path but often in little change in the torsional oscillator response. While the magnitude of the torsional anomaly and phonon mean free path can be very different in different samples, no correlation was found between them.
\end{abstract}

PACS: $\quad 67.80 . b d$ Superfluidity in solid ${ }^{4} \mathrm{He}$, supersolid ${ }^{4} \mathrm{He}$;

65.40.-b Thermal properties of crystalline solids.

Keywords: solid helium, torsional oscillations, shear modulus, phonon mean free path, vibrating dislocations, isotopic impurities.

\section{Introduction}

This paper describes recent experimental investigations of solid helium performed in the Low Temperature Group at Manchester. Some of the techniques used in this research are built upon original advances by Professor Leonid Mezhov-Deglin. It is also delightful to see the continuity of generations of researchers brought up in the Laboratory of Quantum Crystals in ISSP (Chernogolovka) founded by him: A.I.G. worked there in 1981-1992, and M.Yu.B. is currently employed in the Laboratory. We are honoured that this paper appears in the special volume celebrating Leonid Mezhov-Deglin's 75th anniversary.

\section{Solid helium}

Solid helium, thanks to the low mass of atoms and their weak attraction, has a potential to behave very differently from classical crystals. At low temperatures and densities, substantial zero-point kinetic energy and quantum tunneling of atoms lead to a range of unconvential properties, both observed and predicted. The former include freeparticle-like dynamics of point defects (vacancies and impurities) [1,2], multiple exchange causing competing ferroand antiferromagnetic ordering of nuclear spins [3], crystallization waves [4]. Further exotic properties of this quantum crystal might be expected, such as supersolidity 
(coexistence of solid rigidity and non-dissipative coherent mass transport) [1]. Many of the mentioned properties are only meaningful for perfect monocrystals of solid helium. Using the technique to grow large monocrystals of solid helium, developed by Shalnikov [5], Mezhov-Deglin has observed not only ballistic transport of thermal phonons but also hydrodynamics of the phonon gas [6], which, for instance, was a pre-requisite for observations of such a spectacular effect as the second sound (heat propagating as a wave) in solid helium [7].

Disordered samples of solid helium revealed another potential for discoveries. Dislocations were shown to be dynamic [8] and anneal readily [9] at quite low temperatures. Recently, the frequency of torsional oscillations (TO) of polycrystalline solid helium [10] (and a virtually identical effect on its shear modulus [11]), was shown to have temperature dependence below $300 \mathrm{mK}$; this started the new era of interest in solid helium.

It was found that the resonant frequency $f_{r}$ of torsional oscillators, that have solid helium inside the torsion head and channel drilled through the torsion rod, increases by $\Delta f_{r}$ with cooling through some temperature $T^{*}$. There is an associated excess dissipation at $T=T^{*}$. The width of the cross-over in $f_{r}(T)$ is of order temperature $T^{*}$. With increasing the concentration of ${ }^{3} \mathrm{He}$ impurities, $x_{3}$, from below $0.1 \mathrm{ppb}$ to $10 \mathrm{ppm}, T^{*}$ systematically increases from $\sim 20$ to $\sim 200 \mathrm{mK}$ [12]. Increasing frequency of oscillations by a small amount resulted in an increase in $T^{*}$ [13]. The effect was the largest in the most disordered samples, but can be reduced upon annealing and was absent in perfect monocrystals. The shear modulus was found to have the same temperature and frequency dependence — both in solid ${ }^{4} \mathrm{He}$ and ${ }^{3} \mathrm{He}$ samples. It is currently understood that a substantial part of the frequency shift $\Delta f_{r}$ of torsional oscillations is caused by the change in the shear modulus of solid helium [14-17].

It seems crystal defects play a major role in these phenomena. Because of the strong variations of the measured effect between different samples of solid helium, some ways of complementary characterization of samples in situ are required. In this paper, we describe our attempt to combine the torsional oscillator, capable of high-precision measurements at two very different resonant frequencies, with the means of measuring the thermal conductivity of helium samples using the techniques developed by Mezhov-Deglin in the 1960's [6]. At temperatures below $\sim 0.5 \mathrm{~K}$ phonon-phonon interactions become rare, and their mean free path is controlled by scaterings off the crystal boundaries and dislocations. In this context, the mean free path of thermal phonons becomes the measure of the presence of dislocations as seen by high-frequency $\left(10^{9}-10^{10} \mathrm{~Hz}\right)$ oscillations of solid helium. Experimental details and some results were published in $[18,19]$.

\section{Experimental details}

We used an annular cell composed of two coaxial cylindrical stainless steel pieces brazed together at the bottom. The outer and inner walls were 0.3 and $0.5 \mathrm{~mm}$ thick, respectively. The annular gap between the walls was $d=0.3 \mathrm{~mm}$, the outer diameter of the cell was $13 \mathrm{~mm}$ and the height was $14 \mathrm{~mm}$. At the top the cell was brazed to a beryllium copper piece consisting of a disk-shaped plate, a torsion rod ("head rod") and a flange (see Fig. 1). The flange was indium-sealed to a beryllium bronze parallelepiped, capacitively coupled to two flat electrodes (not shown in Fig. 1) for driving and detecting the oscillations, with another torsion rod ("bob rod") that was attached to the platform with an indium seal. A channel drilled through both torsion rods formed the fill line. The oscillator consisting of the cell ("head"), a dummy bob (the electrode with its flange) and the two torsion rods could thus be excited in two torsional modes (symmetric and antisymmetric) with resonant frequencies equal to 132.5 and $853.6 \mathrm{~Hz}$ at $T=300 \mathrm{mK}$. The platform (not shown in Fig. 1) held a heater and two thermometers ( ${ }^{3} \mathrm{He}$ melting curve thermometer and a calibrated germanium resistor) and was thermally connected to the mixing chamber of a dilution refrigerator through a copper wire, $\sim 0.5 \mathrm{~mm}$ in diameter and $\sim 5 \mathrm{~cm}$ in length.

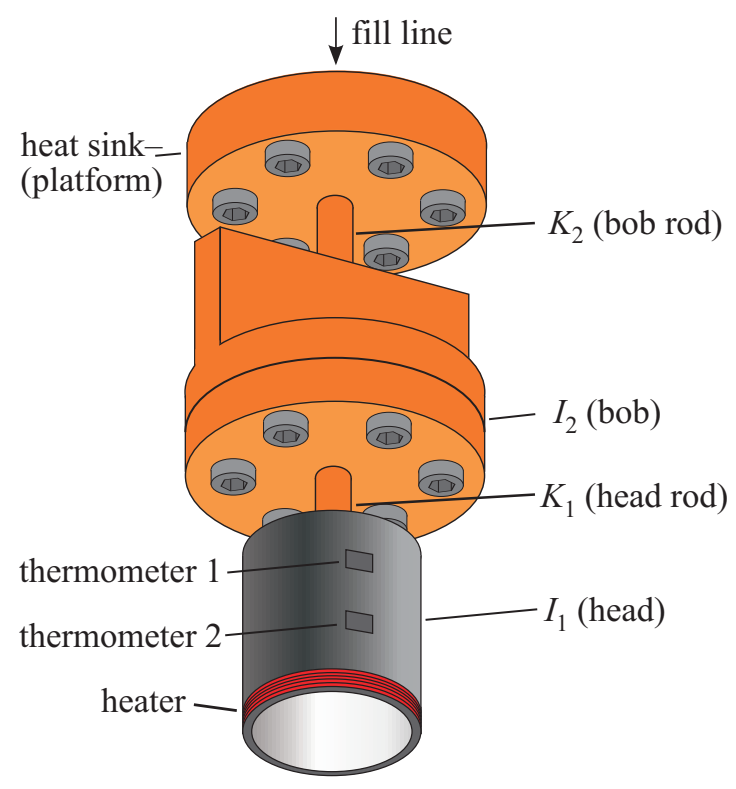

Fig. 1. (Color online) The annular cell with solid helium in which high-quality resonances of torsional oscillations at two different frequencies could be excited and monitored, and the heat flux along the axis could be used to measure the thermal conductivity. The moments of inertia of the annular cell (torsion head) and dummy bob are labelled $I_{1}$ and $I_{2}$, respectively. The rigidity of the "head" torsion rod is $K_{1}$ and that of the "bob" torsion rod is $K_{2}$. The cylindrical channel drilled through both torsion rods and dummy bob allows to fill the cell with helium. 
Thermal conductivity was measured by means of running a known current through the $1.5 \mathrm{k} \Omega$ gradient heater at the bottom of the cell and subsequently measuring the temperature difference established between two $\mathrm{RuO}_{2}$ thick-film resistor thermometers glued with varnish to the side of the cell $7 \mathrm{~mm}$ apart. The temperature of the upper thermometer $T_{1}$ was stabilized by applying a computer-controlled power to the heater attached to the platform. At each value of $T$ we used 4-5 different values of the current in the gradient heater (from zero to maximum compatible with the cooling power of the cryostat). Depending on temperature, the process of stabilization took up to $1 \mathrm{~h}$, after that it could take up to $1 \mathrm{~h}$ to average the readings of the thermometers in the steady state regime. Their temperature difference was proportional to the power dissipated in the gradient heater; the value of $\kappa$ was thus calculated using a known geometrical factor. The typical applied powers ranged from $10 \mathrm{nW}$ to $1 \mu \mathrm{W}$, and typical temperature differences were from 0.1 to $1 \mathrm{mK}$, i.e., within $10^{-3}-10^{-2} \mathrm{~T}$.

In the preliminary experiments we measured the thermal comductivity of the empty cell and it was found to be proportional to temperature and consistent with the data for stainless steel. This contribution did not exceed $20 \%$ of the total thermal conductivity at the lowest temperatures and was subtracted. We have also measured the temperature dependence of the resonant frequencies and the resonant line widths of the empty TO. These were subsequently subtracted from the data obtained with the filled cell. The low and high resonant frequencies of the empty cell increased by 4.5 and $1.0 \mathrm{mHz}$, respectively, upon cooling from 1.2 to $0.02 \mathrm{~K}$.

Samples were grown from either ${ }^{4} \mathrm{He}$, isotopically purified using heat flush technique [20,21] $\left(x_{3} \leq 10^{-10}\right),{ }^{4} \mathrm{He}$ with natural isotopic ratio $\left(x_{3} \sim 3 \cdot 10^{-7}\right)$ or mixture with $x_{3}=2.5 \cdot 10^{-6}$. In all cases the cell was pressurized with liquid ${ }^{4} \mathrm{He}$ to $P=84$ bar at $3.5 \mathrm{~K}$ and then cooled down to form solid at $P=51$ bar and $T=2.35 \mathrm{~K}$ (corresponding to the molar volume $V_{m}=19.5 \mathrm{~cm}^{3}$ and Debye temperature $\left.\Theta_{D}=31.5 \mathrm{~K}[22,23]\right)$. During the cool-down the fill line was first blocked by solid near the $1 \mathrm{~K}$ pot of the dilution refrigerator, and then the sample in the cell grew at constant volume ("blocked capillary method"). The low and high resonant frequencies of the TO decreased by 0.015 and $1.61 \mathrm{~Hz}$ upon filling the cell and solidification. To melt and regrow the sample we used either the gradient heater or the platform heater. By varying the heater current and heating time we were able to vary the sample growth time between $\sim 40 \mathrm{~s}$ and several hours.

\section{Thermal conductivity}

The kinetic theory formula for thermal conductivity [24]

$$
\kappa=\frac{1}{3} C_{V} \bar{v} \ell
$$

where $C_{V} \propto T^{3}$ is the phonon specific heat and $\left.\bar{v}=\left\langle v^{-2}\right\rangle /<v^{-3}\right\rangle$ is the phonon velocity averaged for all branches and crystal orientations (which we approximate by the Debye velocity), allows to determine the phonon mean free path (m.f.p.) $\ell$. This approach was used by Levchenko and Mezhov-Deglin [9] in their studies of recovery of dislocations in plastically-deformed solid helium.

The main result is summarized in Fig. 2. Firstly, the measured temperature dependence of $\kappa(T)$ indeed follows approximately $T^{3}$ dependence between 100 and $300 \mathrm{mK}$; one can thus extract a temperature-independent parameter $\ell$ characterizing the particular sample. Secondly, $\ell$ was generally found to be slightly different for different samples grown in the same conditions; annealing always resulted in the increase of $\ell$ by a factor of order 2. Thirdly, with increasing $x_{3}$ up to $2.5 \mathrm{ppm}, \ell$ steadily increases. These observations allow us to conclude that the phonon scattering cross-section of the defects that determine $\ell$ decreases with annealing and increasing $x_{3}$. The latter points at the role of pinning of dislocations by ${ }^{3} \mathrm{He}$ impurities. The plausible explanation for the increase of $\ell$ upon such pinning is that the resonant scattering of phonons off the vibrating segments of dislocation is mainly responsible for setting the value of $\ell$. Pinning by ${ }^{3} \mathrm{He}$ impurities is known to arrest those vibrations (or shift their frequency to higher values away from the range important for the scattering of thermal phonons at $\sim 100 \mathrm{mK}$ ) and hence increases the relaxation time and mean free path $\ell$.

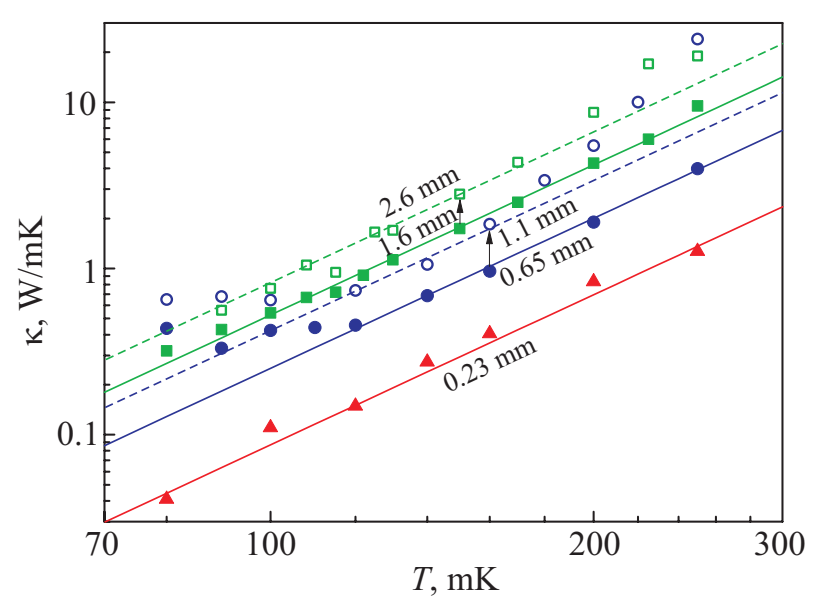

Fig. 2. (Color online) Thermal conductivity of a sample of purified (red $\triangle$ ), $x_{3} \sim 0.3 \mathrm{ppm}$ (blue $\bigcirc$ ) and $x_{3}=2.5 \mathrm{ppm}$ (green $\square$ ). As-grown samples are shown by closed symbols, while those after annealing for $\sim 10 \mathrm{~h}$ at $1.77 \mathrm{~K}$ are shown by open ones (and are connected to their as-grown counterparts by arrows). The lines indicate $T^{3}$ dependence and are labelled by the corresponding values of m.f.p. 


\section{Torsion oscillations results}

The response of the torsional oscillator to AC drive is known to depend on both the inertia and elastic properties of the sample of solid helium inside the torsion head [14-16] and torsion rod [17]. As our oscillator was built in quite a rigid way (thus minimising the differential displacement of its outer and inner walls - and hence the shear deformation of the sample) and has thin-annulus geometry, we expect the shift in the resonant frequency and linewidth to chiefly reflect the sample's effective inertia and perhaps shear modulus of the helium inside the torsion rod (see next Section).

At small amplitudes of AC drive, the response of the torsional oscillator was linear. Typical temperature dependences of the resonant frequency $f_{1}(T)$ are shown in Fig. 3 after subtracting the resonant frequency of the empty cell. The high-temperature contribution of an unknown origin, linear in temperature, is shown by straight lines; in what follows it is subtracted.

In Fig. 4, we compare the temperature dependences of the resonant frequencies $f_{r}(T)$, normalized to their total frequency shift $\Delta f$, for three samples of solid helium with different $x_{3}$. The relevant parameters are listed in Table 1 . One can see that the cross-over temperature $T^{*}$ is not a unique property of a sample but increases with frequency (similar to the dependence of the shear modulus on frequency [11]). With increasing $x_{3}, T^{*}$ generally increases [12]. The logarithmic width of the cross-over, $T_{\mathrm{high}}^{*} / T_{\mathrm{low}}^{*}$ is greater than unity and keeps increasing with increasing $T^{*}$.

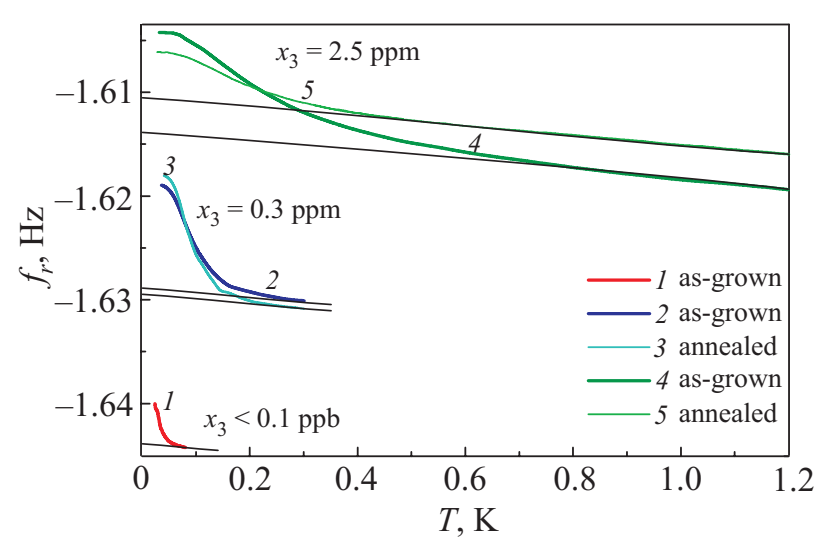

Fig. 3. (Color online) TO resonant frequency $f_{r}(T)$ for samples of different $x_{3}$ with the resonant frequency of an empty cell subtracted. For clarity, the dataset for $x_{3}=0.3 \mathrm{ppm}$ and $x_{3}<0.1 \mathrm{ppb}$ are shifted downwards by 0.015 and $0.030 \mathrm{mHz}$, respectively. The high-temperature contributions, linear in temperature, are shown by straight black lines. They have the same slope obtained by fitting to the high-temperature part of the dataset for the annealed sample with $x_{3}=2.5 \mathrm{ppm}$.

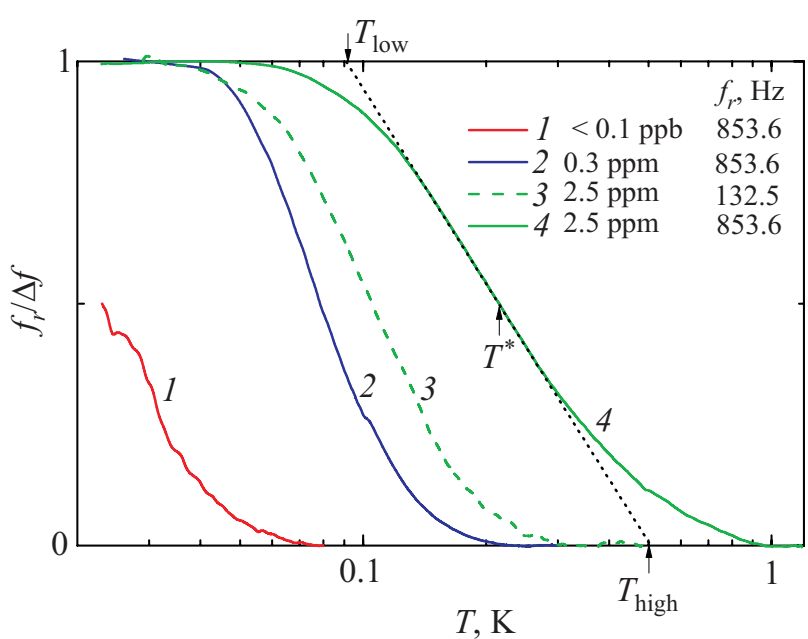

Fig. 4. (Color online) Normalized TO resonant frequency $f_{r} / \Delta f_{r}(T)$ for as-grown samples of different $x_{3}$. For the sample with $x_{3}=2.5 \mathrm{ppm}$, both high-frequency (solid green line) and low-frequency (dashed green line) modes are shown; for others, only the high-frequency mode is presented. The high-temperature components linear in temperature (straight black lines in Fig. 3) are subtracted. An example is given, for the high-frequency dataset for $x_{3}=2.5 \mathrm{ppm}$, of the definitions of the central temperature $T^{*}$ and the width of the cross-over in terms of the low and high temperature limits $T_{\text {low }}$ and $T_{\text {high }}$.

Table 1. Parameters of $f_{r}(T)$ datasets for the three samples from Fig. 4

\begin{tabular}{r|r|r|r|c}
\hline \hline$x_{3}$ & $f_{r}, \mathrm{~Hz}$ & \multicolumn{1}{c|}{$\Delta f_{r}, \mathrm{~Hz}$} & $T^{*}, \mathrm{~K}$ & $T_{\text {high }}^{*} / T_{\text {low }}^{*}$ \\
\hline \hline$<0.1 \mathrm{ppb}$ & 853.6 & $\approx 0.0078$ & $\approx 0.023$ & - \\
$\approx 0.3 \mathrm{ppm}$ & 853.6 & 0.0091 & 0.079 & 2.5 \\
$2.5 \mathrm{ppm}$ & 853.6 & 0.0112 & 0.214 & 5.6 \\
$2.5 \mathrm{ppm}$ & 132.5 & 0.0017 & 0.105 & 3.5 \\
\hline \hline
\end{tabular}

Driving a torsional oscillator at large amplitude is known to suppress the effect [10,25]; we confirm this. On the other hand, we found that the thermal conductivity is insensitive to the amplitude of oscillations. As was noted earlier $[25,26]$, the dependences of the TO resonant frequency on amplitude $V$ look basically identical to the temperature dependences $f_{r}\left(T / T^{*}\right)$, when plotted as $f_{r}\left(\left(V / V^{*}\right)^{\gamma}\right)$, where $\gamma=0.43$. The same exponent $\gamma=0.43$ was observed in comparing the dependences of the shear modulus on temperature, $\mu\left(T / T^{*}\right)$, and strain, $\mu\left(\left(\varepsilon / \varepsilon^{*}\right)^{\gamma}\right)$ $[27,28]$. While we also confirm the close similarity of the curves $f_{r}\left(T / T^{*}\right)$ and $f_{r}\left(\left(V / V^{*}\right)^{\gamma}\right)$ (see Fig. 5), our exponent $\gamma \approx 0.71$ is substantially different.

\section{Inertia or elasticity?}

The original goal of the experiment was to investigate the same sample of polycrystalline solid helium with three techniques: torsional oscillations at a low frequency $f_{2}=132.5 \mathrm{~Hz}$, torsional oscillations at a high frequency 
$f_{1}=853.6 \mathrm{~Hz}$, and thermal conductivity by ballistic phonons (a thermal phonon at $T=200 \mathrm{mK}$ is an oscillating strain with frequency $\sim 4 \mathrm{GHz}$ ). As we will show below, each technique actually investigated a different sample: the low-frequency TO were mainly sensitive to solid helium within the channel drilled inside the "bob rod", the highfrequency TO responded to the properties of solid helium inside the "head rod", while the thermal conductivity was characterizing (as was intended) the sample grown in the annular cavity inside the head of the torsional oscillator.

Our torsional oscillator (see Fig. 1) had two parts ("head" and "bob" with moments of inertia $I_{1}$ and $I_{2}$, respectively). The head is connected to the bob by the torsional rod ("head rod") with rigidity $K_{1}$, and the bob is connected to the rigid and bulky platform by a similar torsional rod ("bob rod") with rigidity $K_{2} . K_{1} \approx K_{2}$ and $I_{1} \ll I_{2}$. In the symmetric mode the deformation of the bottom rod is negligible compared to that of the upper one; the resonant frequency is $f_{2} \approx 1 / 2 \pi\left(K_{2} / I_{2}\right)^{1 / 2}$. In the antisymmetric mode the deformation of the upper rod is negligible compared to that of the bottom one; the resonant frequency is $f_{1} \approx 1 / 2 \pi\left(K_{1} / I_{1}\right)^{1 / 2}$. From the values of these frequencies and assuming $K_{1} \approx K_{2}$, we obtain $I_{2} / I_{1} \approx$ $\approx(853.6 / 132.5)^{2}=41.5$.

Upon filling the empty cell with solid helium, the resonant frequencies have decreased by the following amounts: $\Delta f_{1 s}=-1.61 \mathrm{~Hz}$ and $\Delta f_{2 s}=-0.015 \mathrm{~Hz}$. Adding helium increases the moment of inertia of the head by some $\Delta I_{1 s} \ll I_{1}$; this translates into the expected ratio of frequency shifts $\Delta f_{1 s} / \Delta f_{2 s} \approx\left(I_{2} / I_{1}\right)^{3 / 2}=\left(f_{1} / f_{2}\right)^{3}=267$. Solid helium also increases the rigidity of torsion rods by some small $\Delta K_{1 s} \approx \Delta K_{2 s}$, that would cause an increase in resonant frequencies with their ratio $\Delta f_{1 s} / \Delta f_{2 s} \approx\left(I_{2} / I_{1}\right)^{1 / 2} \approx$ $\approx f_{1} / f_{2}=6.4$. The experimental ratio of frequency shifts, $\Delta f_{1 s} / \Delta f_{2 s}=1.61 / 0.015=107$, is much closer to that expected due to the inertial loading of the torsion head. We thus conclude that it is the inertial contribution which is dominant in the frequency shifts caused by filling the cell.

A very different situation arises after cooling the cell with solid helium through the TO anomaly (say, from $\sim 500$ to $\sim 50 \mathrm{mK}$ for the sample with $x_{3}=2.5 \mathrm{ppm}$ that we investigated). The resonant frequencies increase by $\Delta f_{1}=0.0112 \mathrm{~Hz}$ and $\Delta f_{2}=0.0017 \mathrm{~Hz}$. If one attributes these to the change of the effective inertia then the "superfluid fraction" measured at high frequency, $\Delta f_{1} / \Delta f_{1 s}=0.69 \%$, is very different from that at the low frequency $\Delta f_{2} / \Delta f_{2 s}=12 \%$ ! On the other hand, if we assume that both frequency shifts are caused by the stiffening of solid helium that causes an increase in the rod's rigidities by the same value $\Delta K_{1} \approx \Delta K_{2}$, one expects the ratio of shifts $\Delta f_{1} / \Delta f_{2} \approx f_{1} / f_{2}=6.4$ (see previous paragraph). And indeed, the experimentally measured ratio $\Delta f_{1} / \Delta f_{2}=0.0112 / 0.0017=6.6$ is pretty much the same! We conclude that the TO frequency shift with cooling through $T^{*}$ is caused by the increase in the rigidity of solid helium in torsion rods.

The torsional oscillator was designed in the conventional way that allows to separate the inertial and rigidity elements and their contributions, and hence to use the simple analytical formulae for the resonant frequencies used above. Yet, there is also (negligible) inertia associated with torsional rods and some distributed elasticity of the torsional head and solid helium inside it. In principle, an increase in the shear modulus of solid helium inside the torsion head can also cause an upward frequency shift; these should mainly affect the high-frequency resonance. Experimentally, we found that both resonant frequencies increased by the same fraction when cooled through $T^{*}$; hence, it seems that contributions from the shear modulus of helium inside the torsion head are small compared to those from helium inside TO rods.

The hypothesis that the temperature dependence of the shear modulus of solid helium inside torsion rods is behind the shifts of both resonance frequencies was confirmed by the following observations (using the sample with $x_{3}=2.5 \mathrm{ppm}$, presented in Fig. 5). By increasing the heating of the gradient heater while decreasing that of the platform, we maintained the temperature of the torsion head, $T_{\text {head }}=0.13 \mathrm{~K}$, constant while decreasing that of the mixing chamber, $T_{\text {mixer }}$, from 0.13 to $0.089 \mathrm{~K}$. This resulted in increases of both resonant frequencies $f_{1}$ and $f_{2}$. Then, using the equilibrium temperature dependences $f_{1}(T)$ and $f_{2}(T)$ (measured when the gradient heater was switched off), we can attribute an effective temperature to each frequency shift. It turned out that the effective temperature, $T_{f 2}$, of the low-frequency symmetric resonance was always much closer to $T_{\text {mixer }}$ (namely,

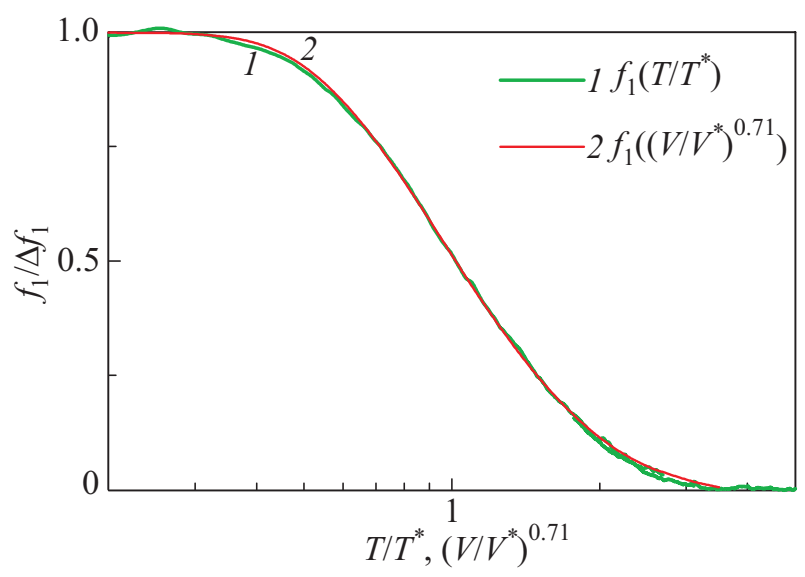

Fig. 5. (Color online) Green line (1) - the shift of the resonant frequency of the asymmetric TO mode $f_{1}$ (at small TO amplitude $V$ ) normalized to the total shift $\Delta f_{1}$ as function of reduced temperature $T / T^{*}$. Red line (2) - the shift of $f_{1}$ (at low temperature $T=0.023 \mathrm{~K}$ ) normalized to the total shift $\Delta f_{1}$ as function of scaled reduced amplitude of oscillations $\left(V / V^{*}\right)^{0.71}$. 
$\left.T_{f 2}-T_{\text {mixer }} \approx 0.15\left(T_{\text {head }}-T_{\text {mixer }}\right)\right)$. On the other hand, the antisymmetric mode had its effective temperature closer to that of the torsion head, $T_{f 1}-T_{\text {mixer }} \approx$ $\approx 0.74\left(T_{\text {head }}-T_{\text {mixer }}\right)$. This confirms that the phenomenon responsible for the symmetric (antisymmetric) resonance is localized in the "bob rod" ("head rod").

Finally, now and then the resonant frequency of the symmetric mode experienced abrupt irreversible shifts while that of the asymmetric mode stayed unchanged. This indicates that these two resonances are sensitive to the state of solid helium in different locations - apparently, inside the two different torsion rods.

\section{Discussion}

We investigated the temperature dependences of three different types of oscillations: torsional oscillations at low frequences 133 and $854 \mathrm{~Hz}$, and high-frequency oscillatory stress (thermal phonons) at frequencies $10^{9}-10^{10} \mathrm{~Hz}$. Their responses to annealing and adding ${ }^{3} \mathrm{He}$ impurities were quite different. The temperature of the TO anomaly moved up upon adding ${ }^{3} \mathrm{He}$; its magnitude and width, while being sample-dependent, did not always decrease upon annealing. The characteristic temperature increased with frequency. The mean free path of thermal phonons, on the other hand, while also sample-dependent, always increased following annealing. It also always increased with increasing the amount of ${ }^{3} \mathrm{He}$.

We argue that, in our experimental set-up, the TO and thermal conductivity probe solid helium at different locations. The absence of correlations between the TO frequency shifts $\Delta f_{r}$ and phonon mean free path $\ell$, that was

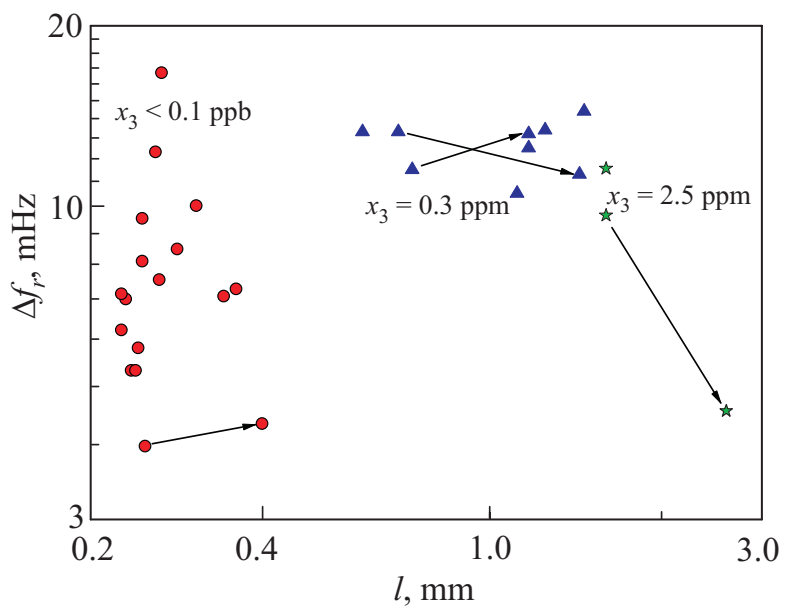

Fig. 6. (Color online) The shift in the TO resonant frequency $\Delta f_{r}$ vs. the phonon m.f.p. for all samples of purified ${ }^{4} \mathrm{He}$ (open circles), well-grade ${ }^{4} \mathrm{He}$ (solid triangles) and mixture with $x_{3}=2.5 \mathrm{ppm}$ (asterisks) studied. As for samples of purified ${ }^{4} \mathrm{He}$, $T^{*} \approx 23 \mathrm{mK}$ (judging by the position of the maximum of the linewidth), we could not reach the limit $T \ll T^{*}$ and hence approximate $\Delta f_{r} \approx 2\left(f_{r}(23 \mathrm{mK})-f_{r}(80 \mathrm{mK})\right)$. Arrows show changes upon annealing at $T=1.77 \mathrm{~K}$. reported previously $[18,19]$ (see Fig. 6) might thus not be too surprising. As there are no evidences that the TO frequency shifts are related to anything but the temperature dependence of the shear modulus of polycrystalline solid helium, in what follows we discuss the observed features in terms of the interactions of dislocations with ${ }^{3} \mathrm{He}$ impurities as a function of temperature.

Annealing at temperature $T=1.8 \mathrm{~K}$ (not too close to the melting point $T_{m}=2.35 \mathrm{~K}$ ) is expected to allow individual dislocations to change configuration in order to reduce the strain energy while perhaps leaving the grain structure of a polycrystal unchanged. It might also help ${ }^{3} \mathrm{He}$ impurities to redistribute around dislocations. There are two very different points of view on the dynamics of ${ }^{3}$ He impurities near dislocations.

If ${ }^{3} \mathrm{He}$ quasiparticles are sufficiently mobile (which is certainly the case at low $x_{3}$ in unstrained regions of crystalline helium), and are hence in thermodynamic equilibrium, their density near dislocation cores reversibly increases with cooling (and the characteristic temperature $T^{*}$ increases with $x_{3}$ ). It is then natural to interpret the stiffening of the shear modulus with cooling as the consequence of pinning dislocations by impurities [11]. Softenning of solid helium caused by large-amplitude oscillations is then associated with stress-induced breakaway of ${ }^{3} \mathrm{He}$ impurities from dislocations. The related nonlinearities, hysteresis and long relaxation times [26] would indicate strong deviations from thermodynamic equilibrium, discussed in the next paragraph. The increase in the phonon mean free path upon either adding ${ }^{3} \mathrm{He}$ or annealing can be explained by reducing the number of dislocation segments capable of vibrating at frequencies of thermal phonons.

Alternatively, if ${ }^{3} \mathrm{He}$ atoms are localized (due to their narrow energy band [2] of $J \sim 10^{-4} \mathrm{~K}$ ) by the stress field in the vicinity of a dislocation (and are only allowed to diffuse slowly via incoherent hopping mediated by phonons), the relaxation time becomes long compared to the experimental time, so the system is permanently out of equilibrium [29]. The dependence of the shear modulus (and of the hypothetical superfluid density also) on temperature and concentartion of impurities $x_{3}$ is explained by the roughening transition of individual dislocations. It is perhaps possible to account for the increase in the phonon mean free path with adding ${ }^{3} \mathrm{He}$ in the framework of this approach but we are unaware of any existing theoretical calculations.

\section{Conclusions}

By combining several experimental techniques we investigated polycrystalline solid ${ }^{4} \mathrm{He}$ with small densities of ${ }^{3} \mathrm{He}$ impurities at temperatures below $1 \mathrm{~K}$. The presence of two torsion rods and a heater on the torsion head allowed to conclude that the temperature-dependent shift of resonant frequency of TO is caused by solid helium not inside 
the torsion head but inside the torsion rods. The temperature-dependent change in the shear modulus of solid helium is the most likely reason for this frequency shift. The dependences of such a shift on the frequency and amplitude of the TO mode and on the concentration of isotopic impurities agree qualitatively with this interpretation. Measurements of the thermal conductivity of solid helium below $0.3 \mathrm{~K}$ resulted in the values of phonon mean free path increasing with increasing the concentration of impurities and with annealing of samples; these point at the rapidly-vibrating dislocation segments, that can be arrested by pinning by point defects, as the dominant scatterers of thermal phonons. More research, both theoretical and experimental, is needed to understand the mechanisms involved in all of the mentioned phenomena. Even though the subject of the dynamics of phonons, point defects and dislocations in solid helium is nearly half-century old, it is still full of promise!

We thank P.V.E. McClintock for providing isotopically purified ${ }^{4} \mathrm{He}$, and R. Schanen for his contribution at the preparatory stage of the experiment, R. Schanen for his contribution at the preparatory stage of the experiment, and S. Gillot and M. Sellers for building the TO cell. This work was supported by the Engineering and Physical Sciences Research Council [grant number EP/H014691].

1. A.F. Andreev and I.M. Lifshitz, Sov. Phys. JETP 29, 1107 (1969).

2. V.N. Grigoriev, B.N. Eselson, and V.A. Mikheev, Zh. Eksp. Teor. Fiz. 64, 608 (1973).

3. M. Roger, J.H. Hetherington, and J.M. Delrieu, Rev. Mod. Phys. 55, 1 (1983).

4. K.O. Keshishev, A.Ya. Parshin, and A.B. Babkin, Pis'ma Zh. Eksp. Teor. Fiz. 30, 63 (1979) [JETP Lett. 30, 516 (1979)].

5. A.I. Shal'nikov, Zh. Eksp. Teor. Fiz. 41, 1056 (1961) [Sov. Phys. JETP 14, 753 (1962)].

6. L.P. Mezhov-Deglin, Zh. Eksp. Teor. Fiz. 49, 66 (1965) [Sov. Phys. JETP 22, 47 (1966)].

7. C.C. Ackerman, B. Bertman, H.A. Fairbank, and R.A. Guyer, Phys. Rev. Lett. 16, 789 (1966).

8. V.L. Tsymbalenko, Sov. Phys. JETP 60, 537 (1984).
9. A.A. Levchenko and L.P. Mezhov-Deglin, Zh. Eksp. Theor. Fiz. 86, 2123 (1984); [Sov. Phys. JETP 59, 1234 (1984)].

10. E. Kim and M.H.W. Chan, Nature (London) 427, 225 (2004).

11. O. Syshchenko, J. Day, and J. Beamish, Phys. Rev. Lett. 104, 195301 (2010).

12. E. Kim, J.S. Xia, J.T. West, X. Lin, A.C. Clark, and M.H.W. Chan, Phys. Rev. Lett. 100, 065301 (2008).

13. Y. Aoki, J.C. Graves, and H. Kojima, Phys. Rev. Lett. 99, 015301 (2007).

14. D.Y. Kim and M.H.W. Chan, arXiv:1207.7050v1.

15. J.D. Reppy, X. Mi, A. Justin, and E.J. Mueller, J. Low Temp. Phys. 168, 175 (2012).

16. H.J. Maris, Phys. Rev. B 86, 020502 (2012).

17. J.R. Beamish, A.D. Fefferman, A. Haziot, X. Rojas, and S. Balibar, Phys. Rev. B 85, 180501 (2012).

18. D.E. Zmeev and A.I. Golov, Phys. Rev. Lett. 107, 065302 (2011).

19. D.E. Zmeev, M.Yu. Brazhnikov, R. Schanen, and A.I. Golov, J. Low Temp. Phys. (2012), DOI 10.1007/s10909-0120665-9.

20. P.C. Hendry and P.V.E. McClintock, Cryogenics 27, 131 (1987).

21. P.V.E. McClintock (private communication) While the heatflush technique [20] is capable of producing ${ }^{4} \mathrm{He}$ with an undetectably low concentartion of ${ }^{3} \mathrm{He}$, the resulting gas might get contaminated when in contact with gas-handling manifolds containing tiny residual level of ${ }^{3} \mathrm{He}$. Recent mass-spectrometer characterization of four samples of isotopically-purified ${ }^{4} \mathrm{He}$ resulted in three samples showing anomalously high ${ }^{3} \mathrm{He}$ concentration of $x_{3} \sim 10^{-10}$ and only one having $x_{3}<10^{-12}$.

22. A. Driessen, E. van der Poll, and I.F. Silvera, Phys. Rev. B 33, 3269 (1986).

23. D.S. Greywall, Phys. Rev. B 16, 5127 (1977).

24. J.M. Ziman, Electrons and Phonons, Oxford U. P. (1960).

25. B. Hunt, E. Pratt, V. Gadagkar, M. Yamashita, A.V. Balatsky, and J.C. Davis, Science 324, 632 (2009).

26. E.J. Pratt, B. Hunt, V. Gadagkar, M. Yamashita, M.J. Graf, A.V. Balatsky, and J.C. Davis, Science 332, 821 (2011).

27. J. Day and J. Beamish, Nature 450, 853 (2007).

28. J. Day, O. Syshchenko, and J. Beamish, Phys. Rev. Lett. 104, 075302 (2010).

29. D. Aleinikava and A.B. Kuklov, J. Low Temp. Phys. (2012), DOI 10.1007/s10909-012-0646-z. 